\title{
Decoding Arm Movements by Myoelectric Signal and Artificial Neural Networks
}

\author{
Alexandre Balbinot, Adalberto Schuck Júnior, Gabriela Winkler Favieiro \\ Department of Electrical Engineering, Federal University of Rio Grande do Sul, Porto Alegre, Brazil \\ Email: alexandre.balbinot@ufrgs.br, aulaeng@gmail.com
}

Received September 22, 2012; revised October 22, 2012; accepted October 30, 2012

\begin{abstract}
The scientific researches in the field of rehabilitation engineering are increasingly providing mechanisms in order to help people with disability to perform simple tasks of day-to-day. Several studies have been carried out highlighting the advantages of using muscle signal in order to control rehabilitation devices, such as experimental prostheses. This paper presents a study investigating the use of forearm surface electromyography (sEMG) signals for classification of several movements of the arm using just three pairs of surface electrodes located in strategic places. The classification is done by an artificial neural network to process signal features to recognize performed movements. The average accuracy reached for the classification of six different movements was $68 \%-88 \%$.
\end{abstract}

Keywords: Surface Electromyography (sEMG); Artificial Neural Network (ANN); Assistive Technology; Prosthesis

\section{Introduction}

Researches in Biomedical Engineering and Computational Intelligence are providing mechanisms to help people with some disabilities to perform simple tasks of day-today [1-4]. The development of systems managed by myoelectric signals with the intention to reproduce the human arm movement is far from perfect, which makes it the target of many investigations [5-16]. In recent years, there has been an explosion of interest in computational intelligence $(\mathrm{CI})$ as evidenced by the numerous applications in health, biomedicine, and biomedical engineering. CI techniques are computing algorithms and learning machines, including artificial neural networks, fuzzy logic, genetic algorithms, and support vector machines [1-13]. Develop a robotic prosthesis as similar as possible to a human arm is not a simple task. There is great difficulty both in the area to distinguish the various degrees of freedom that the arm may have as in the development of a robotic prosthesis that can perform all these movements.

The myoelectric signal is the sign of muscle control of the human body that contains the information of the user's intent to contract a muscle and, therefore, make a move. Studies shows that the Amputees are able to generate standardized myoelectric signals repeatedly before of the intention to perform a certain movement [5-7]. It makes the use of this signal very advantageous, because the control of a robotic prosthesis can be performed based in the intention of the user.

Many studies are being conducted in able-bodies sub- jects to verify the feasibility and performance of different algorithms for pattern recognition using EMG signals from the forearm muscles [5-15]. In these studies are usually employed a high number of electrode pairs, ranging for 4 to 12. Using classification patterns techniques such as LDA [5], fuzzy logic [4,7-9,15], artificial neural networks [16], among others, was found high accuracies $(>90 \%)$ for the classification of different moves [4-10]. This suggests that it is possible to achieve high accuracy using several pairs of electrodes.

A recent study estimated that for 12 pairs of electrodes was possible to classify 10 different movements with an accuracy of $81.2 \%$ and decreasing the number of pairs of electrode to eight, the classification accuracy dropped 3\% [5]. The control of the prosthesis can be accomplished in several ways. One form of control is performed by a set of electrodes to picking up signals of different muscles, and depending on the muscle that the patient has contracted, it should result in a certain movement of the prosthesis $[13,14]$.

Shenoy presented a study using surface electromyography in real time to control a robotic arm using electrodes in the arm, at eight sites chosen carefully, using only the effective value and continuing qualification of a window of data signal. An accuracy of $92 \%-98 \%$ was obtained at eight different movements (opening and closing the hand, wrist flexion and extension movement, right to left wrist and arm rotation). It is also possible to control the prosthesis through the recognition of electromyographic signals using an artificial neural network 
(ANN), linking these patterns with the moves that the prosthesis hould accomplish [16]. In this case, the number of electrodes can be optimized.

It is important to notice that almost all of the previous studies used at least 4 pairs of electrodes to evaluate the performance of the pattern recognition algorithms. The proposed system uses only 3 pairs of electrodes for signals acquisition, and processes these signals with an artificial neural network for recognition of performed movements, including more than one movement performed at the same time. In this context, the objective of this work is to evaluate the performance of an artificial neural network model for recognition of performed movements, including more than one movement performed at the same time.

\section{Materials and Methods}

\section{1. sEMG Data Acquisition}

Figure 1 shows the block diagram of the experiment developed for the acquisition of myoelectric signals, as well as for the characterization of signals indicative of movements of hand-arm segment.

The system includes an electromyography (EMG) of 8 channels used for the capture of myoelectric signals using surface electrodes and, through an acquisition board (NI USB-6009, 14 bit sampling rate of $1 \mathrm{kHz}$ - data acquisition for each $1 \mathrm{~ms}$ was sufficient to identify the moves being made by volunteers), the signal is digitized and inserted into a computer, where it will be filtered, processed and analyzed by software, using the technique of artificial neural networks to characterize the movements. The acquisition of signals is carried out through bipolar surface electrodes with passive configuration. The choice of recording sites for muscle activity is motivated by the relevance of the chosen muscles to the gestures must be classified. For this study, the captured signals belong to three muscles: Flexor Carpi Ulnaris (channel 1), Extensor Carpi Radialis Longus (channel 2) and Biceps (channel 3) - in this case, using three channels of EMG (electromyography) - see Figure 2. The proposed system is based on using myoelectric signals of only a few muscles, but performs a complex signal processing for the characterization of the movements-see Figure 3.

\section{2. sEMG Preprocessing and Feature Extraction}

The data was collected from two able-bodies subjects over three consecutive trials: calibration, adaptation and performance test. The calibration is an important step because its aims to check if the electrodes are positioned correctly and also to determine a threshold value that will be used later to detect the occurrence of a movement. This procedure involves capturing the muscle signal during one second at a time of relaxation and in a moment of maximum voluntary contraction (MVC). If one of the electrode's pairs were not correctly positioned, the signal received will have low quality and would be necessary to do the reposition of the electrodes until the signal to noise ratio reaches at least a rate greater than 10 , based on the value established in tests of the signal acquisition previously performed. A percentage ranging from $15 \%$ to $30 \%$ of the average peak values, acquired from the MVC movement, is also used as threshold which indicated whether occurred or not a muscle contraction.

The adaptation's trial has the function to adapt the system for each subject, once it depends on the variability due to different muscle activities that each person can make. The trial consists in a session with five repetitions for each chosen movement to train the pattern classification algorithm.

In order to verify the feasibility and accuracy of the system is accomplished the performance test. The sEMG data were collect over 5 sessions. A session consists in five repetitions of each of the six determined moves, in random order. It's represents a total of 150 movements.

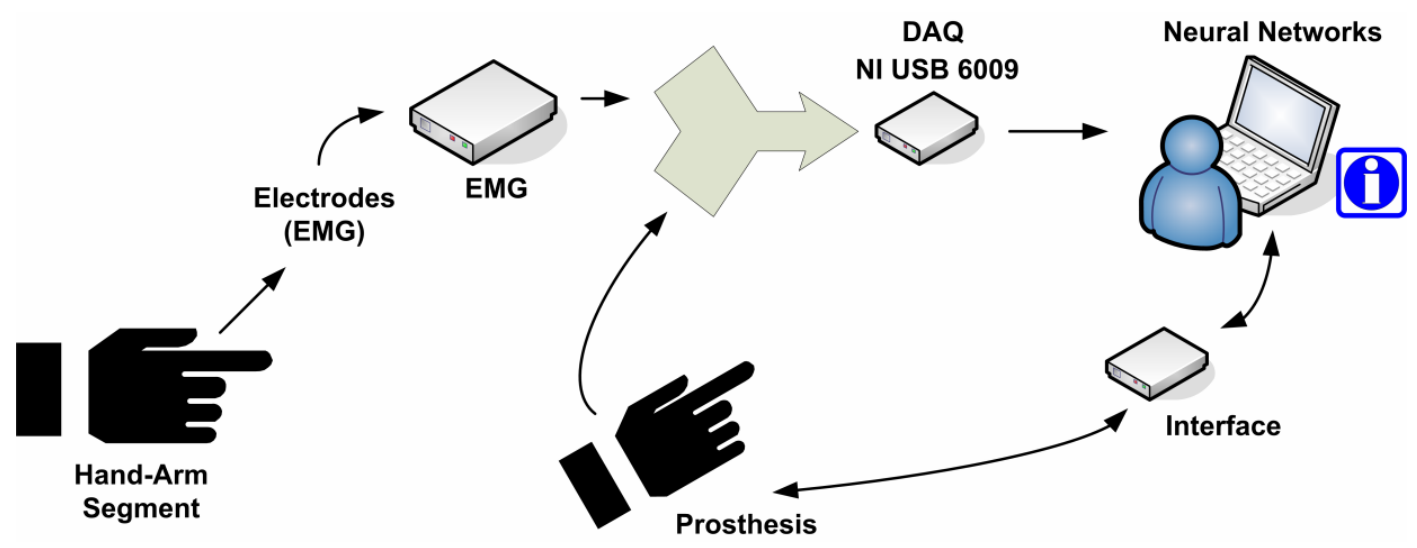

Figure 1. The proposed system diagram blocks. 


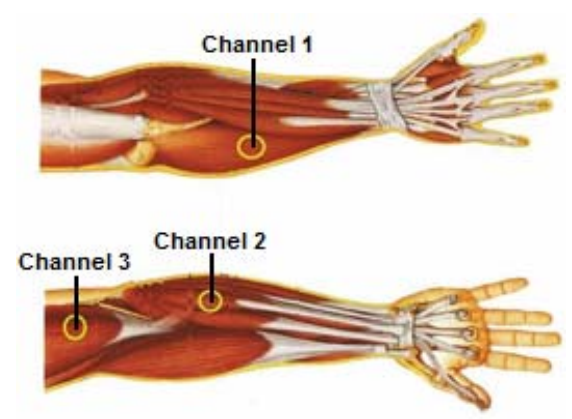

Figure 2. Non-invasive placement of electrodes.

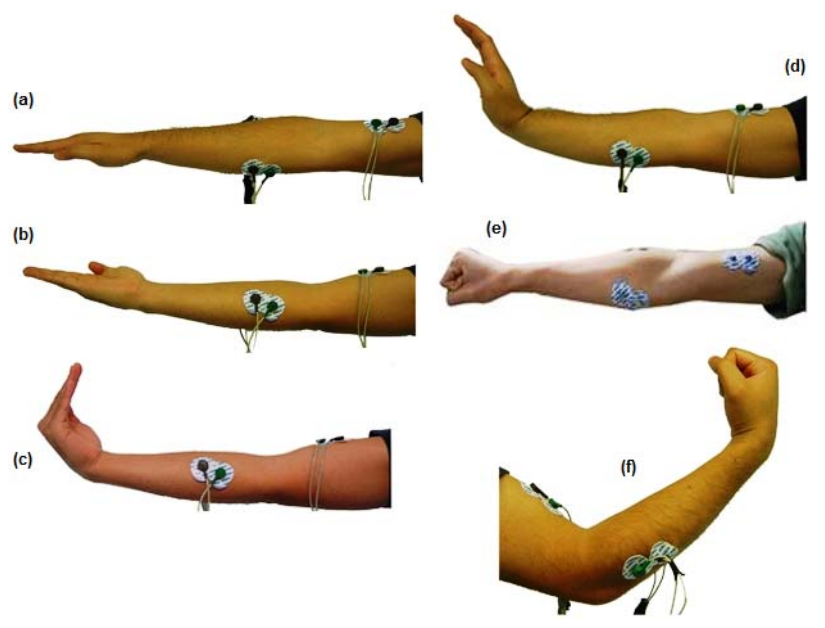

Figure 3. Pictures of the movements characterized by the developed system: (a) Relaxed; (b) Forearm rotation; (c) Wrist flexion; (d) Wrist extension; (e) Hand contraction and (f) Forearm flexion.

For every trial, demonstrations of each movement were shown in a LCD screen and subjects were instructed to perform the movements that appear in random order, as shown in Figure 4. Between consecutives movements was determined an interval ranging from 3 - 5 seconds. Figure 5 shows the signal for the three channels of EMG during the tests.

\subsection{Prosthesis Prototype}

A prototype of a mechanical prosthesis of the segment hand-arm connected between the interface circuit (motor drive) and the computer was developed to carry out the tests - see Figure 1. Figure 6 shows the design of the arm developed for this application. The design of the prosthesis was developed in the CAD Solid Edge ST to reproduce a human arm, the most similar as possible. It was designed a robotic arm with four degrees of freedom: flexion and extension of the forearm, rotation of the arm, flexion and extension of the wrist and open and close the hand. The prosthesis robotics has about $40 \mathrm{~cm}$ long and 8 $\mathrm{cm}$ wide, size that was considered adequate for the proposed system.

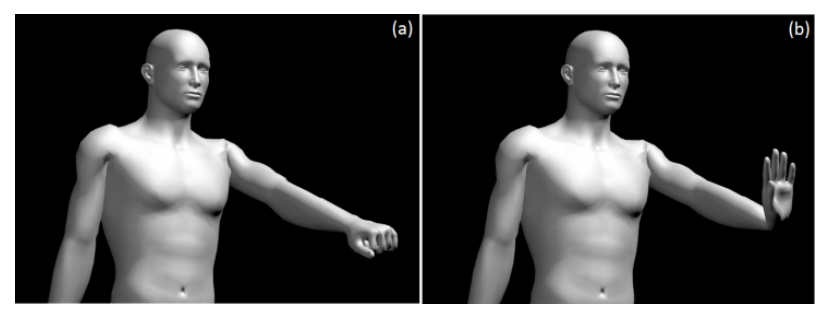

Figure 4. Pictures of the movements of the virtual arm interface: (a) Hand contraction and (b) Wrist extension.

Besides the development of each piece of robotic prostheses, was also performed in Solid Edge tool, the simulation of the degree of rotation that each board would have to be prototyped. The board representing the elbow, responsible for flexion and extension of the forearm, has $90^{\circ}$ rotation. The board representing the handle has $180^{\circ}$ rotation. The joint of forearm rotation has rotation of $360^{\circ}$, but for that, the joint is limited by software to be as anthropomorphic as possible. The joints of the hand, representing the phalanxes of the fingers, have $90^{\circ}$ rotation each. The material chosen to construct the prosthesis was Technyl ( ${ }^{\circ}$ Rhodia), because it presents the following main features: low specific weight, high wear resistance and abrasion resistance, high melting point, high resistance to chemical agents, self-extinguibilidade flame, self-lubricating, absorption of vibration, high shock resistance, low coefficient of friction and mechanical movements silent, among others.

\subsection{Classification with Artificial Neural Network}

The myoelectric signal processing was accomplished through an artificial neural network. This network received the pre-processed rms value of each channel of data acquisition. After all the processing, this network produced outputs that characterized movements being made by the human arm. The selected topology of the artificial neural network to implement the recognition of complex movements is the network of multiple layers perceptions (MLP). The MLP typically consists of a set of sensory units that constitute the input layer, one or more hidden layers of computational nodes and one output layer. The input signal is propagated forward through the network, layer by layer. Multilayer networks can use a large number of learning techniques. For this application, it was selected the back propagation algorithm. In this case, was applied to the ANN an array of pre-determined entry and were analyzed the response of the network through the values of the output layer, which were compared with the desired response to compute the value of the error function. In turn, the error signals were propagated back through the network, against the direction of synaptic connections. The synaptic weights were adjusted, so that the actual response of network moved closer to the desired response 

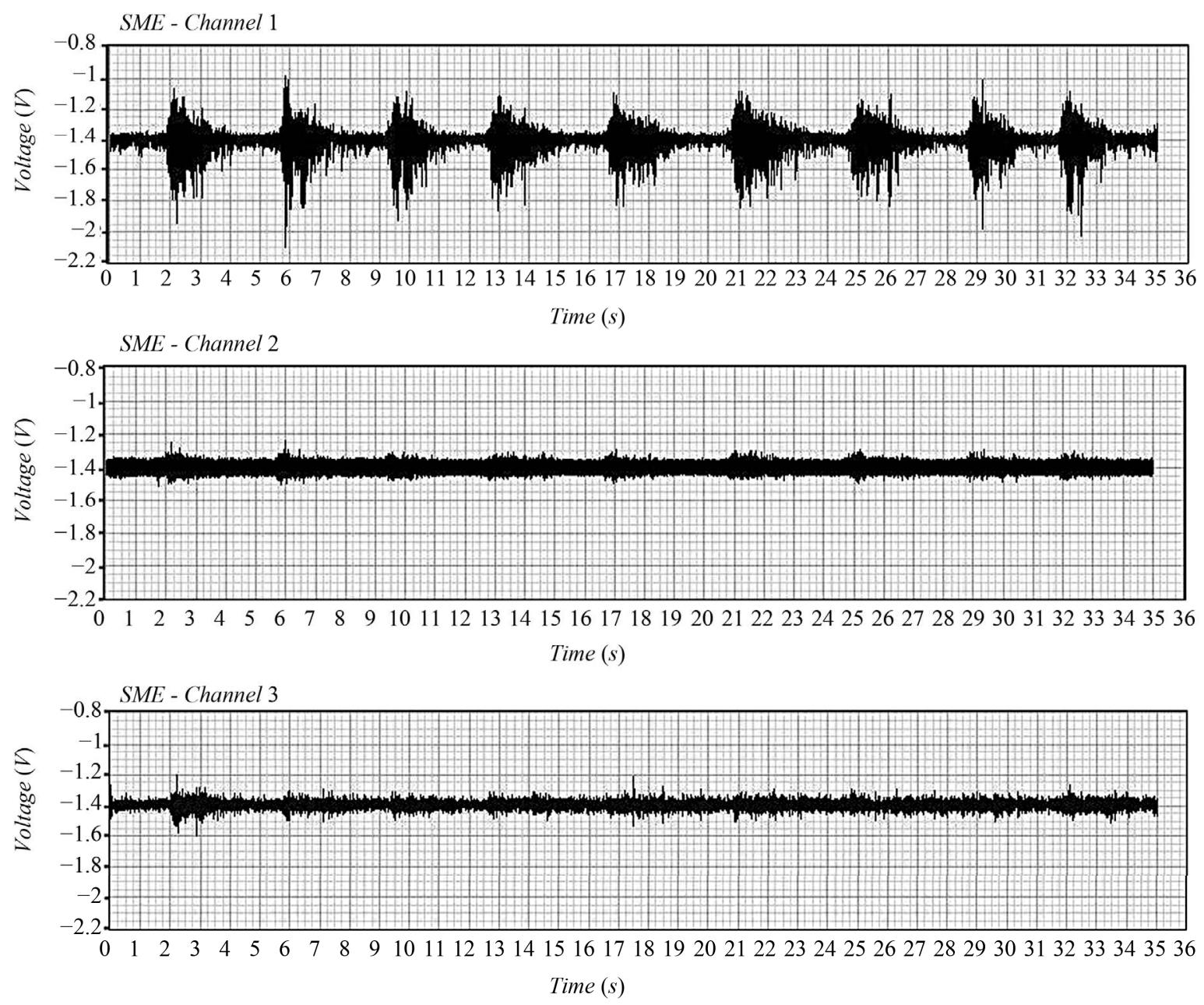

Figure 5. Signal acquisition during motion of wrist extension.

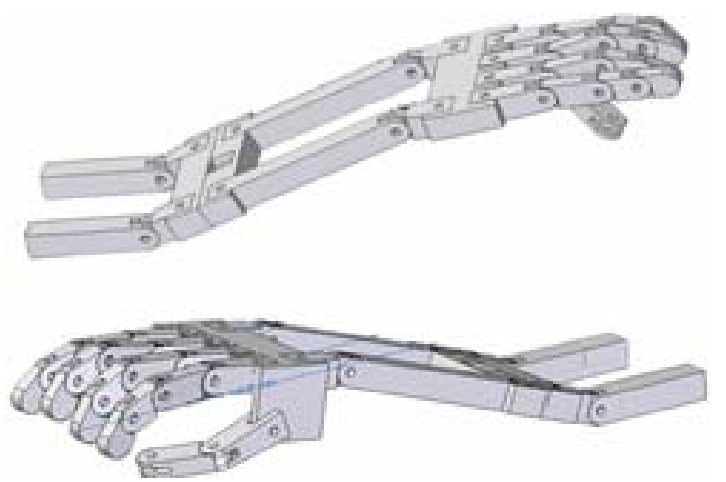

Figure 6. Lateral view of the robotic prosthesis.

in a statistical sense.

The ANN developed has two dimensions of hidden layers with ten neurons each, with three entries placed in the network (rms value of each channel) and five outputs are generated that represent the basic moves that experimental prosthesis can perform (contraction of the hand, wrist extension, wrist flexion, forearm flexion and forearm rotation). The function used for the hidden layers of the network is called sigmoid (tansig), and generates values in a range of -1 to 1 . However, the output layer of ANN uses a linear function for the network training and also for the use of neural network, was used the routine of windowing the signal, in which the rms value of the windowed signal was used as input to the network. The only difference is that, for the training was provided the expected result for that particular input, and, the result was equal to 1 if there was an occurrence of certain movements and 0 if not. The output of the ANN had generated infinite values, because the output layer used a linear function, so was considered that a certain movement occurs only when the corresponding output value is greater than 0.8 .

\subsection{Experimental Statistical Analysis}

Many experiments involve more than two factors. For statistical validation methodology was used the "Design and analysis of three-factor experiments-Three-Factor Fixed Effects Model". Consider the three-factor-factorial experiment, with underlying model Equation (1): 


$$
\begin{aligned}
Y_{i j k l}= & \mu+\tau_{i}+\beta_{j}+\gamma_{k}+(\tau \beta)_{i j}+(\tau \gamma)_{i k}+(\beta \gamma)_{j k} \\
+ & +(\tau \beta \gamma)_{i j k}+\epsilon_{i j k l}\left\{\begin{array}{l}
i=1,2, \cdots, a \\
j=1,2, \cdots, b \\
k=1,2, \cdots, c \\
l=1,2, \cdots, n
\end{array}\right.
\end{aligned}
$$

where $\mu$ is the overall mean effect, $\tau_{i}$ is the effect of the $i$ th level of factor $A$ (three different muscles or three channels $1-3), \beta_{j}$ is the effect of the $j$ th level of factor $B$ (two subjects: 1 and 2), $\gamma_{k}$ is the effect of the $k$ th level of factor $C$ (five movements: wrist flexion, wrist extension, hand contraction, forearm flexion, forearm rotation, forearm rotation and hand contraction), $(\tau \beta)_{i j}$ is the effect of the interaction between $A$ and $B,(\tau \gamma)_{i k}$ is the effect of the interaction between $A$ and $C,(\beta \gamma)_{j k}$ is the effect of the interaction between $B$ and $C,(\tau \beta \gamma)_{i j k}$ is the effect of the interaction between $A, B$ and $C$ and $\varepsilon_{i j k l}$ is a random error component having a normal distribution with mean zero and variance $\sigma^{2}$. Notice that the model contains three main effects ( $A, B$ and $C$ ), three two-factor interactions, a three-factor interaction, and an error term. This experimental design is a completely randomized design.

\section{Results and Discussion}

\subsection{Data Collection}

The data was collected from two subjects over five sessions, as mentioned earlier. A session consists by five repetitions of the specified movements. The subjects were instructed to relax between the movements and maintain each gesture comfortably. There wasn't any restriction or measure about the force exerted by the subjects during arm movements.

The classification of each movement occurred during the data acquisition. The signal was acquired during the following simple movements: contraction of the hand, wrist extension, wrist flexion, forearm flexion and forearm rotation. Was also carried out an assay for the acquisition of a complex movement: forearm rotation along with the movement of contraction of the hand. For training the neural network was used one session for each move with 5 repetitions, and the training time lasted less than 1 second. The training uses the Levemberg-Marquardt algorithm and for the calculation of performance was used the technique of the mean squared error. The training was made for the two subjects separately. The subject one reached the mean squared error of 0.0165 as shown in Figure 7 and the subject 2 achieved an error of 0.0531 .

\subsection{Statistical Analysis}

The $F$-test on main effects and interactions follows directly from the expected mean squares. These ratios fol-

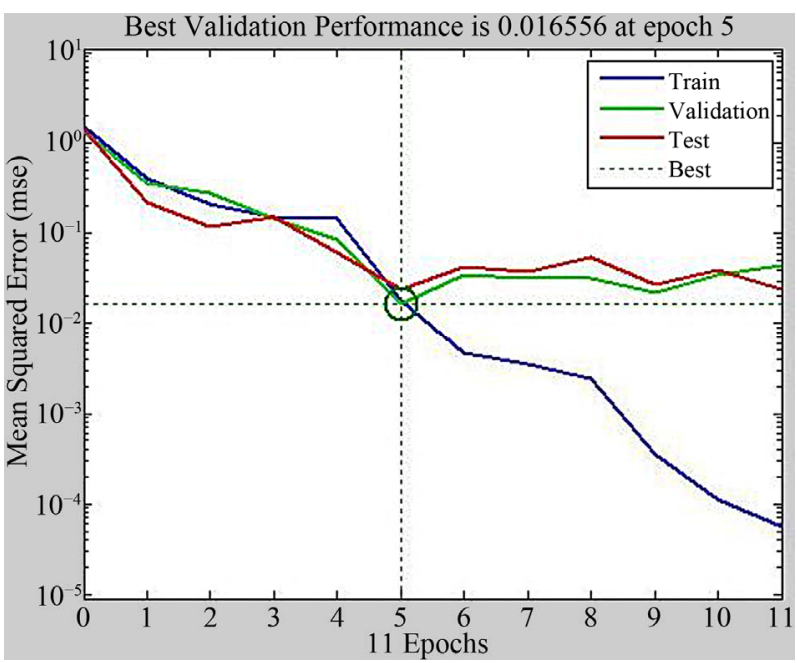

Figure 7. Graph of the mean squared error generated by the training of the neural network for the subject one.

low $F$ distributions under the respective null hypotheses. We will use $\alpha=0.05$ (significance level). The analysis of variance for a three-factor experiment showed that the main effects due to the three channels, two subjects and five movements are significant, in other words. Thus, it is possible to say that the output rms for each one of the three channels, two subjects and five movements are quite distinct from each other, and thus, the myoelectric signals are also distinct and so can be treated as distinct channels by the developed neural networks model.

The results of this model showed that the interactions are true, ie $(\tau \beta),(\tau \gamma),(\beta \gamma)$ and $(\tau \beta \gamma)$ are significant. However, the ANOVA doesn't identify which means are different. Methods for investigating this issue are called multiple comparisons methods. In this study we used the Fisher's least significant difference (LSD) method. From this analysis, we see that there are significant differences between all pairs of means.

\subsection{Classification Accuracy}

The signal processing was performed with two individuals, to verify the difference in system performance. The two individuals participated in a same process of training and system testing. Thus it was standardized that the training stage would receive five repetitions for each movement and the step test would receive twenty and five repetitions. The average accuracy reached for the classification of six different movements was $68 \%$ - $88 \%$. It's shown in the Table 1 and Figure 8 the results acquired for each subject.

The system showed a good performance in the use of few electrodes. The subject two has shown a worse performance than the subject one. It can happen due the fact that occurred some difficult in order to position the electrodes and the subject proved tired in the last part of the 
Table 1. Results for subject 1 and subject 2.

\begin{tabular}{cccc}
\hline & & Subject 1 & \\
\cline { 2 - 4 } Movements & Repetitions & Hits & Accuracy (\%) \\
\hline Wrist flexion & 25 & 25 & 100 \\
Wrist extension & 25 & 18 & 72 \\
Hand contraction & 25 & 25 & 100 \\
Forearm flexion & 25 & 25 & 100 \\
Forearm rotation & 25 & 25 & 100 \\
Forearm rotation and hand contraction & 25 & 14 & 56 \\
TOTAL & 150 & 132 & $\mathbf{8 8}$ \\
Movements & & Subject 2 & Accuracy (\%) \\
Wrist flexion & Repetitions & Hits & 64 \\
Wrist extension & 25 & 16 & 60 \\
Hand contraction & 25 & 15 & 84 \\
Forearm flexion & 25 & 21 & 60 \\
Forearm rotation & 25 & 15 & 92
\end{tabular}

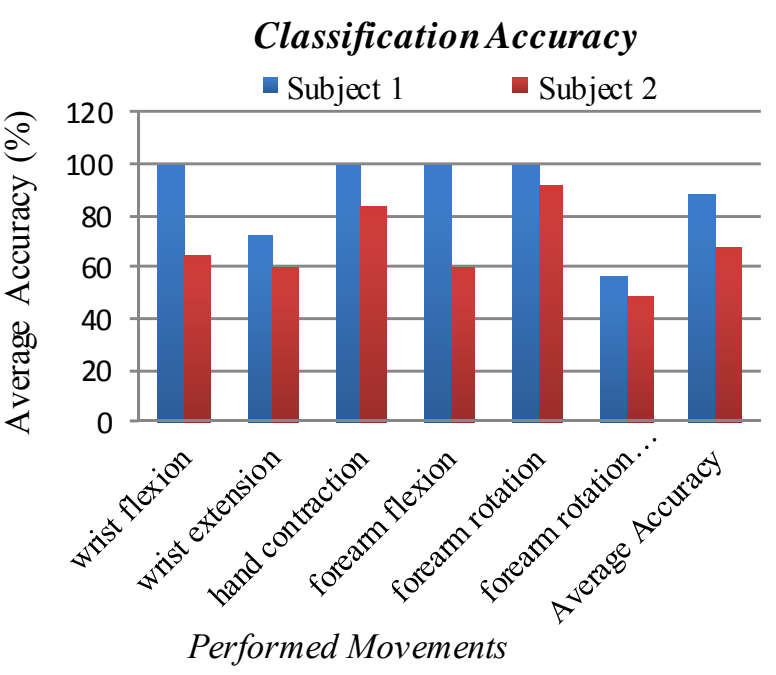

Figure 8. Achieved results in terms of classification accuracy.

assay. The subject moved a lot because of the incorrect posture in the chair and because the repetitive effort realized. Another possible cause for the difference of the results is the lost of the efficiency of the contact electrode-skin, decreasing the signal amplitude. Another important thing is that the delay on the system is of just 200 $\mathrm{ms}$, the time of the signal processing.

\section{Conclusions}

The proposed system had the purpose to use a limited number of acquisition channels of myoelectric signal and with the help of a robust digital signal processing verify the validity of its performance. When tested, the mean peak signal with a maximum voluntary contraction (MVC) performed at least 10 times greater than the mean peak signal muscle at a time of relaxation. Thus, it was possible define a threshold ranging from $15 \%$ to $30 \%$ of MVC to differentiate an occurrence of a muscle contraction, representing a movement. With the windowing of the signal at the moment that occurred a move, was possible to obtain the rms value for each of the three channels and use these values as input to a neural network of 2 hidden layers with 10 neurons per layer. This network aimed to characterize the movements that are running. As can be noticed in the results, some movements have achieved a lower hit rate, this may occur due to poor signal quality and the quantity of motion that was presented to the ANN, since some movements had the answer in terms of rms value very similar.

The proposed system achieved a less average accuracy than the other studies cited in this paper [4-16], however, were used only three pairs of electrodes. But this paper verified the performance achieved when the subject realized more than one movement at the same time, which increased the rate error. It is important to notice that almost all proposed studies do not verify the performance of simultaneous movements of the arm. One possible proposal for future works would be investigate other techniques that could improve the performance of neural network, making the system able to characterize a wide range of complex movements. A practice used to im- 
prove the characterization of the movements would be increase the number of acquisition channels of myoelectric signal and, consequently, increase the amount of captured muscle signals, so that there will be a greater differentiation between the movements executed.

\section{REFERENCES}

[1] Y. Wang, X. Gao, C. Jia, B. Hong and S. Gao, "BrainComputer Interfaces Based on Visual Evoked Potentials," IEEE Engineering in Medicine and Biology Maganize, Vol. 27, No. 5, 2008, pp. 64-71. doi:10.1109/MEMB.2008.923958

[2] M. Hamel, R. Fontaine and P. Boissy, "In-Home Telerehabilitation for Geriatric Patients," IEEE Engineering in Medicine and Biology Maganize, Vol. 27, No. 4, 2008, pp. 29-37. doi:10.1109/MEMB.2008.919491

[3] L. Y. Deng, C. L. Hsu, T. C. Lin, J. S. Tuan and S. M. Chang, "EOG-Based Human-Computer Interface System Development," Expert Systems with Applications, Vol. 37, No. 4, 2010, pp. 3337-3343. doi:10.1016/j.eswa.2009.10.017

[4] A. Balbinot and M. M. G. Barreto, "Modeling Movement of a Hand Prosthesis with Fuzzy Inference Systems," ISSNIP Proceedings of Biosignals and Biorobotics Conference, Australian Research Council/ISSNIP-ARC Research Network on Intelligent Sensors, Sensor Network and Information Processing (ISSNIP), 2010, pp. 283-288.

[5] G. Li, A. E. Schultz and T. A. Kuiken, "Quantifying Pattern Recognition-Based Myoeletric Control of Multifunctional Transradial Prosthesis," IEEE Transactions Neural System Rehabilitation Engineering, Vol. 18, No. 2, 2010, pp. 185-192. doi:10.1109/TNSRE.2009.2039619

[6] K. Momen, S. Krishnan and T. Chau, "Real-Time Classification of Forearm Electromyographic Signals Corresponding to User-Selected Intentional Movements for Multifunction Prosthesis Control," IEEE Transactions Neural System Rehabilitation Engineering, Vol. 15, No. 4, 2007, pp. 535-542. doi:10.1109/TNSRE.2007.908376

[7] A. B. Ajiboye and R. F. Weir, "A Heuristic Fuzzy Logic Approach to EMG Pattern Recognition for Multifunctional Prosthesis Control," IEEE Transactions Neural
System Rehabilitation Engineering, Vol. 13, No. 3, 2005, pp. 280-291. doi:10.1109/TNSRE.2005.847357

[8] F. H. Y. Chan, Y. S. Yang, F. K. Lam, Y. T. Zhang and P. A. Parker, "Fuzzy EMG Classification for Prosthesis Control," IEEE Transactions Rehabilitation Engineering, Vol. 8, No. 3, 2000, pp. 305-311. doi:10.1109/86.867872

[9] B. Karlik, M. O. Tokhi and M. Alci, “A Fuzzy Clustering Neural Network Architecture for Multifunction UpperLimb Prosthesis," IEEE Transactions Biomedical Engineering, Vol. 50, No. 11, 2003, pp. 1255-1261. doi:10.1109/TBME.2003.818469

[10] J. G. Hincapie and R. F. Kirsch, "Feasibility of EMGBased Neural Network Controller for an Upper Extremity Neuroprosthesis," IEEE Transactions Biomedical Engineering, Vol. 17, No. 1, 2009, pp. 80-90.

[11] A. D. Chan and K. B. Englehart, "Continuous Myoelectric Control for Powered Prostheses Using Hidden Markov Models," IEEE Transactions Biomedical Engineering, Vol. 52, Vo. 1, 2005, pp. 121-124.

[12] J. W. Sensinger, B. A. Lock and T. A. Kuiken, “Adaptative Pattern Recognition of Myoeletric Signals: Exploration of Conceptual Framework and Practical Algorithms," IEEE Transactions Biomedical Engineering, Vol. 17, No. 3, 2009, pp. 270-278.

[13] K. Englehart and B. Hudgins, "A Robust, Real-Time Control Scheme for Multifunction Myoelectric Control," IEEE Transactions Biomedical Engineering, Vol. 50, No. 7, 2003, pp. 848-854. doi:10.1109/TBME.2003.813539

[14] B. Hudgins, P. Parker and R. Scott, "A New Strategy for Multifunction Myoelectric Control," IEEE Transactions Biomedical Engineering, Vol. 40, No. 1, 1993, pp. 82-94. doi:10.1109/10.204774

[15] R. N. Khushaba, A. Al-Ani and A. Al-Jumaily, "Orthogonal Fuzzy Neighborhood Discriminant Analysis for Multifunction Myoeletric Hand Control," IEEE Transactions Biomedical Engineering, Vol. 57, No. 6, 2010, pp. 1410-1419. doi:10.1109/TBME.2009.2039480

[16] P. Shenoy, K. J. Miller, B. Crawford and R. P. N. Rao, "Online Electromyographic Control of a Robotic Prosthesis," IEEE Transactions Biomedical Engineering, Vol. 55, No. 3, 2008, pp. 1128-1135. doi:10.1109/TBME.2007.909536 